02

\title{
Функция распределения ионов по скоростям в собственном газе при криогенных газовых температурах
}

\author{
(C) А.С. Мустафраев, ${ }^{1}$ В.О. Некучаев, ${ }^{2}$ В.С. Сухомлинов ${ }^{3,4}$ \\ ${ }^{1}$ Санкт-Петербургский горный университет, \\ 199106 Санкт-Петербург, Россия, \\ 2 Ухтинский государственный технический университет, \\ 169300 Ухта, Респ. Коми, Россия \\ ${ }^{3}$ Санкт-Петербургский государственный университет, физический фракультет, \\ 198504 Санкт-Петербург, Россия \\ ${ }^{4}$ Университет ИТМО, \\ 197101 Санкт-Петербург, Россия \\ e-mail: alexmustafaev@yandex.ru
}

(Поступило в Редакцию 7 июня 2016 г.)

Рассмотрено влияние упругого рассеяния на функцию распределения ионов в плазме собственного газа при малых полях. Получено аналитическое выражение, справедливое и в области криогенных температур атомов. С учетом упругих столкновений проведены расчеты зависимости приведенной подвижности ионов $\mathrm{He}^{+}-\mathrm{He}, \mathrm{Ar}^{+}-\mathrm{Ar}$ в пределе нулевого поля от температуры атомов в диапазоне от 4 до $1000 \mathrm{~K}$, которые хорошо согласуются с имеющимися экспериментальными данными. Показано, что упругие столкновения играют существенную роль в формировании функции распределения ионов при низких температурах. Результаты измерений подвижности ионов в пределе нулевого поля при низких температурах могут быть использованы для получения данных об отношении сечений упругого рассеяния и резонансной перезарядки.

DOI: 10.21883/JTF.2017.03.44236.1915

\section{Введение}

Ранее нами рассмотрено влияние упругих столкновений на дрейф ионов в плазме собственного газа в сильных полях $[1,2]$. Выяснено, что упругие столкновения слабо влияют на дрейф ионов вдоль поля и существенно сказываются на характеристиках их дрейфа поперек направления электрического поля. Это объяснялось тем, что в сильных полях, когда средние энергии ионов велики по сравнению с температурой атомов, сечение упругого рассеяния оказывается намного меньше сечения резонансной перезарядки и, таким образом, этот вид столкновений незначительно влияет на подвижность ионов. В то же время из-за упругих столкновений (хотя и достаточно редких) часть энергии, которую ион приобретает в электрическом поле между столкновениями, переходит в энергию движения иона в плоскости, ортогональной электрическому полю. Это оказывает решающее влияние на рост коэффициента поперечной диффузии ионов с полем, который при отсутствии упругих столкновений определяется температурой атомов. Как известно, сечение упругого рассеяния растет с уменьшением относительной энергии сталкивающихся частиц намного быстрее, чем сечение резонансной перезарядки [3-5]. Для малых полей, когда энергия ионов порядка средней тепловой энергии атомов, при низкой температуре газа сечения могут быть одного порядка $[5,6]$. Таким образом, влияние упругих столкновений при слабых полях на функцию распределения ионов (ФРИ) может быть весьма значительным.
С физической точки зрения влияние упругих столкновений на ФРИ при малых полях в собственном газе вполне понятно. Действительно, в этих условиях ФРИ близка к максвелловской. Наличие слабого электрического поля и резонансной перезарядки приводит к тому, что ФРИ становится анизотропной (вытянутой в направлении поля) и величина этого возмущения зависит от энергии иона по некоторому закону [6,7]. Упругие столкновения, которые происходят с передачей энергии во все направления движения иона, очевидно, приводят к изотропизации ФРИ, т.е. к уменьшению возмущения максвелловского распределения.

Дрейф ионов в собственном газе при малых полях теоретически изучался многими авторами [8-11], результаты подробно описаны в монографии [11]. Большинство из этих исследований основывались на различных приближениях теории Чепмена-Энскога [12] для дрейфовой скорости или подвижности в пределе нулевого поля.

ФРИ в собственном газе при малых полях исследовалась рядом авторов [6,7]. Однако в первой работе учет упругих столкновений ограничивался оценкой их влияния при комнатных температурах, а во второй вообще не проводился. При этом в работе [7] сечение резонансной перезарядки полагалось постоянным. Как будет показано в дальнейшем, для низких температур атомов учет упругих столкновений важен при расчете ФРИ в собственном газе.

Рассмотрим задачу нахождения ФРИ в плазме собственного газа в слабом поле с учетом зависимости сечений резонансной перезарядки и упругого рассеяния от относительной энергии иона и атома. 


\section{Получение основных соотношений}

Для решения поставленной задачи необходимо выбрать модель дифференциального сечения рассеяния при столкновении иона с собственным атомом, учитывающую резонансную перезарядку и упругое рассеяние. В ряде работ указывалось, что изотропное в системе центра масс сечение упругого рассеяния хорошо описывает рассматриваемую ситуацию $[3,5,13]$. Тогда в качестве дифференциального сечения рассеяния выберем функцию $\sigma_{\theta}(\varepsilon, \theta)$ :

$$
\sigma_{\theta}(\varepsilon, \theta)=\frac{1}{2 \pi \sin \theta} \sigma_{b}(\varepsilon) \delta(\theta-\pi)+\frac{\sigma_{i}(\varepsilon)}{4 \pi},
$$

где $\varepsilon, \theta-$ энергия относительного движения в системе центра масс и угол рассеяния в этой системе отсчета соответственно, $\sigma_{b}(\varepsilon)$ - так называемое сечение обратного рассеяния, $\sigma_{i}(\varepsilon)-$ сечение изотропного рассеяния. Отметим, что сечение $\sigma_{b}(\varepsilon)$ не является сечением резонансной перезарядки. Величины $\sigma_{b}(\varepsilon)$ и $\sigma_{i}(\varepsilon)$ найдем, используя известные из экспериментов и (или) квантово-механических расчетов диффузионное сечение (или сечение переноса импульса) $\sigma_{m}(\varepsilon)$ и сечение переноса энергии (или вязкостное сечение) $\sigma_{v}(\varepsilon)$ :

$$
\begin{gathered}
\sigma_{m}(\varepsilon)=2 \pi \int_{0}^{\pi} \sigma_{\theta}(\varepsilon, \theta)(1-\cos \theta) \sin \theta d \theta=2 \sigma_{b}(\varepsilon)+\sigma_{i}(\varepsilon), \\
\sigma_{v}(\varepsilon)=2 \pi \int_{0}^{\pi} \sigma_{\theta}(\varepsilon, \theta)\left(1-\cos ^{2} \theta\right) \sin \theta d \theta=\frac{2}{3} \sigma_{i}(\varepsilon) .
\end{gathered}
$$

Учитывая, что с высокой степенью точности [11]

$$
\sigma_{m} \cong 2 \sigma_{c},
$$

где $\sigma_{c}-$ сечение резонансной перезарядки, из (2) имеем

$$
\sigma_{i}=1.5 \sigma_{v}, \quad \sigma_{b}=\sigma_{c}-0.5 \sigma_{i} .
$$

Таким образом, сечение обратного рассеяния $\sigma_{b}$ равно сечению резонансной перезарядки при $\sigma_{i}=0$.

Рассмотрим установившееся распределение ионов по скоростям при условиях:

- атомы газа движутся в соответствии с законом Максвелла;

- движение ионов происходит в собственном газе с низкой степенью ионизации;

- доминирующими процессами, формирующими ФРИ по скоростям, являются резонансная перезарядка и упругие столкновения ионов с атомами;

- плазма является однородной;

- средняя энергия ионов близка к средней тепловой энергии атомов.

В стационарной плазме при сделанных предположениях уравнение Больцмана имеет вид

$$
\frac{e E}{m} \nabla_{v}\left(f_{i}\right)=S_{i}
$$

где $e, m-$ заряд и масса иона, $\mathbf{E}-$ напряженность электрического поля, $f_{i}-$ ФРИ по скоростям, $S_{i}-$ интеграл столкновений, который можно разделить на два члена $S_{c i}$ и $S_{e i}$. Первый член соответствует обратному рассеянию (в системе центра масс), а второй изотропному (также в системе центра масс) рассеянию иона на собственном атоме. Рассмотрим сначала величину $S_{c i}$. Учитывая, что ион, возникающий в результате перезарядки, имеет скорость атома, определим интеграл столкновений следующим образом $[2,6]$ :

$$
\begin{aligned}
S_{c i}\left(\mathbf{v}_{i}\right)= & n_{a}\left\{f_{a}\left(\mathbf{v}_{i}\right) \int \sigma_{b} v_{r} f_{i}\left(\mathbf{v}_{i}^{\prime}\right) d \mathbf{v}_{i}^{\prime}\right. \\
& \left.-f_{i}\left(\mathbf{v}_{i}\right) \int \sigma_{b} v_{r} f_{a}\left(\mathbf{v}_{a}^{\prime}\right) d \mathbf{v}_{a}^{\prime}\right\} \equiv S_{1 c i}-S_{2 c i},
\end{aligned}
$$

где $\mathbf{v}_{i}-$ скорость иона, $\mathbf{v}_{a}-$ скорость атома, $f_{a}\left(\mathbf{v}_{a}\right)-$ максвелловская функция распределения атомов по скоростям (нормирована на единицу), $v_{r}$ - модуль относительной скорости иона и атома перед столкновением; ФРИ нормирована на концентрацию. Как известно, сечение резонансной перезарядки $\sigma_{c}$ в диапазоне энергий до нескольких электронвольт слабо зависит от относительной энергии иона и атома. В работах $[1,2]$ было показано, каким образом из ФРИ с постоянным значением $\sigma_{c}$ можно получить решение уравнения Больцмана, учитывающее такую зависимость. Поэтому сначала решим поставленную задачу для постоянного сечения резонансной перезарядки, а затем сформулируем правила учета зависимости $\sigma_{c}$ от относительной энергии сталкивающихся частиц.

В работах $[1,14]$ показано, что с точностью до переобозначений выражения для $S_{1 c i}\left(\mathbf{v}_{i}\right), S_{2 c i}\left(\mathbf{v}_{i}\right)$ имеют вид

$$
\begin{gathered}
S_{1 c i}\left(\mathbf{v}_{i}\right)=n_{a} f_{a}\left(\mathbf{v}_{i}\right) \int \sigma_{b} v_{r} f_{i}\left(\mathbf{v}_{i}^{\prime}\right) d \mathbf{v}_{i}^{\prime}, \\
S_{2 c i}\left(\mathbf{v}_{i}\right)=n_{a} \sigma_{b} f_{i}\left(\mathbf{v}_{i}\right) w_{0 e}\left(v_{i}\right) ; \\
w_{0 e}\left(v_{i}\right)=\left(v_{i}+\frac{1}{2 \beta v_{i}}\right) \operatorname{erf}\left(\sqrt{\beta} v_{i}\right)+\frac{\exp \left(-\beta v_{i}^{2}\right)}{\sqrt{\pi \beta}} .
\end{gathered}
$$

Здесь $\operatorname{erf}(x)=\frac{2}{\sqrt{\pi}} \int_{0}^{x} \exp \left(-t^{2}\right) d t, \beta=\frac{1}{2 k T_{a}}, w_{0 e}\left(v_{i}\right)$ - относительные скорости иона и атома, усредненные по функции распределения атомов, $k, T_{a}-$ постоянная Больцмана и температура атомов соответственно.

Рассмотрим теперь интеграл столкновений $S_{e i}$, соответствующий изотропному в системе центра масс рассеянию иона на собственном атоме. Столкновения, происходящие с отклонением иона на малые углы, вызванные взаимодействием заряда иона с наведенным электрическим моментом атома и происходящие при больших прицельных параметрах, слабо влияют на ФРИ, поскольку происходят с малым изменением скорости [15], поэтому мы их не будем учитывать. Кроме того, при небольших энергиях ионов может происходить поляризационный захват [11]. При этом соответствующее дифференциальное сечение, по-видимому, также близко к изотропному в системе центра масс. 
Тогда, принимая во внимание, что перед столкновением атом движется с произвольной скоростью $\mathbf{v}_{a}$, плотность вероятности $g_{e}\left(\mathbf{v}_{i}^{\prime} \rightarrow \mathbf{v}_{i} ; \mathbf{v}_{a}\right)$ того, что ион, имея скорость $\mathbf{v}_{i}^{\prime}$, приобретет в результате столкновения с атомом скорость $\mathbf{v}_{i}$, можно записать в следующем виде [14]:

$$
g_{e}\left(\mathbf{v}_{i}^{\prime} \rightarrow \mathbf{v}_{i} ; \mathbf{v}_{a}\right)=\frac{v_{r}\left(v_{i}^{\prime}\right)}{2 \pi} \delta\left(\xi \mathbf{v}_{a}-\frac{\xi^{2}}{2}-\frac{v_{i}^{2}-v_{i}^{\prime 2}}{2}\right),
$$

где $\xi=\mathbf{v}_{i}-\mathbf{v}_{i}^{\prime} ; \xi=\left|\mathbf{v}_{i}-\mathbf{v}_{i}^{\prime}\right|$.

Для $S_{e i}$ имеем

$$
\begin{aligned}
& S_{e i}\left(\mathbf{v}_{i}, \mathbf{v}_{a}\right)=n_{a} \int \sigma_{i}\left(v_{r}\left(v_{i}^{\prime}\right)\right) v_{r}\left(v_{i}^{\prime}\right) g_{e}\left(\mathbf{v}_{i}^{\prime} \rightarrow \mathbf{v}_{i} ; \mathbf{v}_{a}\right) \\
& \quad \times f_{a}\left(\mathbf{v}_{a}\right) f_{i}\left(\mathbf{v}_{i}^{\prime}\right) d \mathbf{v}_{i}^{\prime}-n_{a} \int \sigma_{i}\left(v_{r}\left(v_{i}\right)\right) v_{r}\left(v_{i}\right) \\
& \quad \times g_{e}\left(\mathbf{v}_{i} \rightarrow \mathbf{v}_{i}^{\prime} ; \mathbf{v}_{a}\right) f_{a}\left(\mathbf{v}_{a}\right) f_{i}\left(\mathbf{v}_{i}\right) d \mathbf{v}_{i}^{\prime} .
\end{aligned}
$$

Интегрируя (8a) по $\mathbf{v}_{a}$ c учетом слабой зависимости сечения $\sigma_{i}\left(v_{r}\left(v_{i}^{\prime}\right)\right)$ от скорости по сравнению с максвелловской функцией, получим [14]

$$
\begin{aligned}
\bar{S}_{e i}\left(\mathbf{v}_{i}\right)= & n_{a} \int \sigma_{i}\left(w_{0 e}\left(v_{i}\right)\right) w_{e}\left(\mathbf{v}_{i}^{\prime} \rightarrow \mathbf{v}_{i}\right) f_{i}\left(\mathbf{v}_{i}^{\prime}\right) d \mathbf{v}_{i}^{\prime} \\
& -n_{a} \sigma_{i}\left(w_{0 e}\left(v_{i}\right)\right) w_{0 e}\left(v_{i}\right) f_{i}\left(\mathbf{v}_{i}\right),
\end{aligned}
$$

где

$$
w_{e}\left(\mathbf{v}_{i}^{\prime} \rightarrow \mathbf{v}_{i}\right)=\frac{\beta}{\pi^{1.5}} \frac{\exp \left[-\beta\left(\frac{v_{i}^{2}-v_{i}^{\prime 2}}{2 \xi}+\frac{\xi}{2}\right)^{2}\right]}{\xi} .
$$

Вводя безразмерную скорость $x=v_{i} \sqrt{\beta}, \beta=m / 2 k T_{a}$, запишем проинтегрированное по $\mathbf{v}_{a}$ уравнение (5) в виде

$$
\begin{aligned}
& \varepsilon_{0} \mu \frac{\partial f_{i}}{\partial x}+2 \tilde{w}_{o e}(x)\left[1+\frac{\sigma_{i}\left(w_{0 e}(x)\right)}{2 \sigma_{c}}\right] f_{i} \\
& =2\left[1-\frac{\sigma_{i}\left(w_{0 e}(x)\right)}{2 \sigma_{c}}\right] f_{a}(x) \int x_{r} f_{i}\left(\mathbf{x}^{\prime}\right) d \mathbf{x}^{\prime} \\
& +\frac{2}{\sigma_{c}} \int \sigma_{i}\left(\tilde{w}_{o e}\left(\mathbf{x}^{\prime}\right)\right) \tilde{w}_{e}\left(\mathbf{x}^{\prime} \rightarrow \mathbf{x}\right) f_{i}\left(\mathbf{x}^{\prime}\right) d \mathbf{x}^{\prime},
\end{aligned}
$$

где $\varepsilon_{0}=\frac{e E}{n_{a} \sigma_{c} k T_{a}} \ll 1, \mu=\cos \theta, \theta-$ угол между скоростью иона и вектором электрического поля, $\tilde{w}_{o e}(x)=$ $=\sqrt{\beta} w_{0 e}(x / \sqrt{\beta}), \quad \tilde{w}_{e}\left(\mathbf{x}^{\prime} \rightarrow \mathbf{x}\right)=\beta^{1.5} w_{e}\left(\mathbf{x}^{\prime} / \sqrt{\beta} \rightarrow \mathbf{x} / \sqrt{\beta}\right)$. Как показано в $[1,2]$, при аппроксимации сечения перезарядки формулой [12]

$$
\sigma_{c}\left(E_{c}\right)=\sigma_{0 c}\left[1+a \ln \left(E_{c}(\mathrm{eV})\right)\right]^{2},
$$

где $E_{c}-$ энергия относительного движения иона и атома. Учет слабой зависимости сечения перезарядки от относительной скорости иона и атома с достаточной степенью точности сводится к замене сечения $\sigma_{c}$ на $\sigma_{0 c} k(x)$, где

$$
k(x)=\left[1+a \ln \left(\frac{x_{0}^{2}}{w_{0 e}(x)^{2}}\right)\right]^{2}
$$

а безразмерная скорость $x_{0}$ соответствует энергии относительного движения иона и атома $1 \mathrm{eV}$. Окончательно имеем

$$
\begin{aligned}
\varepsilon_{0} \mu & \frac{\partial f_{i}}{\partial x}+2 \tilde{w}_{o e}(x)\left[k(x)+0.5 \sigma k_{e}(x)\right] f_{i} \\
= & 2\left[k(x)-0.5 \sigma k_{e}(x)\right] f_{a}(x) \int x_{r} f_{i}\left(\mathbf{x}^{\prime}\right) d \mathbf{x}^{\prime} \\
& +2 \sigma \int k_{e}\left(x^{\prime}\right) \tilde{w}_{e}\left(\mathbf{x}^{\prime} \rightarrow \mathbf{x}\right) f_{i}\left(\mathbf{x}^{\prime}\right) d \mathbf{x}^{\prime},
\end{aligned}
$$

где параметр $\varepsilon_{0}$ определяется для энергии $1 \mathrm{eV}$, $k_{e}(x)=\frac{\sigma_{i}\left(\tilde{w}_{o e}(x)\right)}{\sigma_{i}\left(\tilde{w}_{o e}\left(x_{0}\right)\right)}, \sigma=\frac{\sigma_{i}\left(\tilde{w}_{o e}\left(x_{0}\right)\right)}{\sigma_{0 c}}-$ отношение сечения упругого рассеяния и резонансной перезарядки при энергии относительного движения в системе координат центра масс, равной $1 \mathrm{eV}$.

Так как при малых электрических полях ФРИ слабо анизотропна, то для решения уравнения (11b) применим метод сферических гармоник [14]. Единственное усложнение состоит в том, что оно представляет собой сингулярно возмущенное интегродифференциальное уравнение с малым параметром $\varepsilon_{0}$ при старшей производной $[16,17]$. В Приложении 1 получено его решение в виде

$$
f_{i}(x, \mu)=f_{a}(x)+3 \varepsilon_{0} \mu F_{11}(x),
$$

где $F_{11}(x)$ определена формулами (П5).

Для подвижности в пределе нулевого поля имеем, используя (П5):

$$
K\left[\mathrm{~cm}^{2} / \mathrm{Vc}\right]=\frac{6.98 \cdot 10^{4}}{\sigma_{0 c}\left(10^{-15} \mathrm{~cm}^{-2}\right) \sqrt{T_{a}(K) m}} \int_{0}^{\infty} x^{3} F_{11}(x) d x,
$$

где $m$ - масса иона в атомных единицах.

\section{Обсуждение полученных результатов}

Как уже говорилось, дрейф ионов в собственном газе в слабых полях исследовался многими авторами. Сравним наши результаты с некоторыми из них. В [6] и [12] было получено, что без учета упругих столкновений дрейфовая скорость ионов в этом случае выражается соотношениями соответственно

$$
\frac{0.341 E}{N \sqrt{T_{a} m} \sigma\left(2.12 \sqrt{\frac{2 T_{a}}{m}}\right)} \quad \text { и } \frac{0.332 E}{N \sqrt{T_{a} m} \sigma\left(2.24 \sqrt{\frac{2 T_{a}}{m}}\right)} .
$$

Полученное с использованием $F_{11}^{(2)}(x)$ аналогичное соотношение дает численный коэффициент в этой формуле, равный 0.343, который слабо зависит от температуры атомов и параметра $a$ в (11a).

На рис. 1 приведены результаты сравнения поправки к максвелловской ФРИ $g_{i}(x)=2 \pi F_{11}^{(i)}(x)_{\sigma=0}, i=1,2$, рассчитанной по полученным соотношениям в предположении постоянного сечения перезарядки $(a=0)$ и отсутствия упругих столкновений, и аналогичных функций 


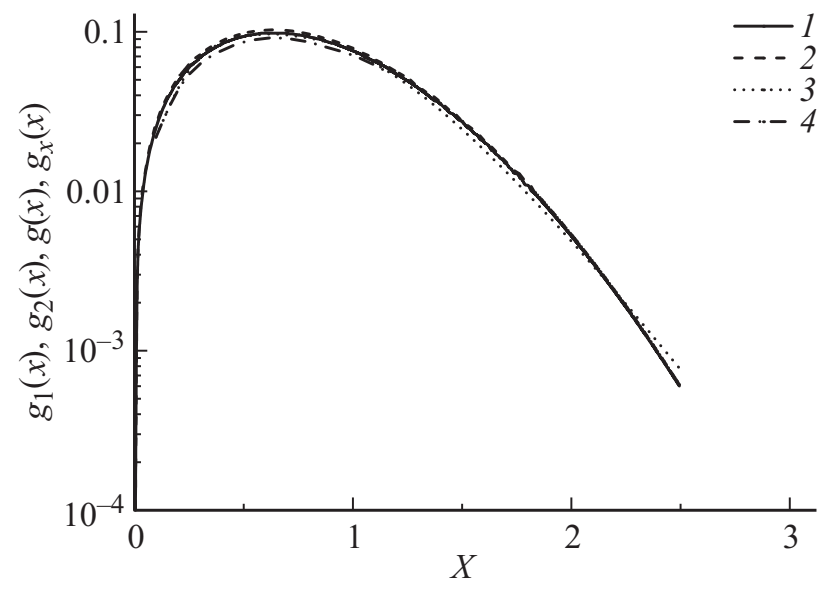

Рис. 1. Возмущения максвелловской ФРИ без учета упругих столкновений в слабом поле при постоянном сечении резонансной перезарядки: $1-g_{1}(x)-$ первое приближение, $2-$ $g_{2}(x)$ - второе приближение, $3-g(x)$ - приближенное решение [6], $4-g_{x}(x)-$ решение [7].

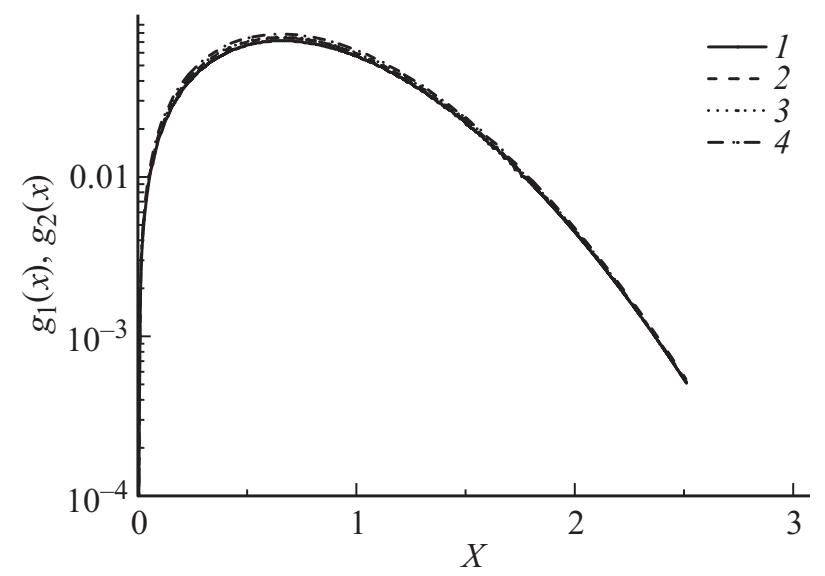

Рис. 2. Возмущения максвелловской ФРИ без учета упругих столкновений в слабом поле: $1-g_{1}(x)-$ первое приближение для случая $\mathrm{He}^{+}-\mathrm{He}, 2-g_{2}(x)-$ второе приближение для случая $\mathrm{He}^{+}-\mathrm{He}, 3-g_{1}(x)-$ первое приближение для случая $\mathrm{Xe}^{+}-\mathrm{Xe}, 4-g_{1}(x)-$ первое приближение для случая $\mathrm{Xe}^{+}-$Хе. Параметры сечений резонансной перезарядки взяты из [18].

$g(x), g_{x}(x)$, вычисленных в [6,7] соответственно. Видно, что, во-первых, эти функции близки, во-вторых, первое приближение $g_{1}(x)$ практически не отличается от второго $g_{2}(x)$ (см. Приложение 1 ). Таким образом, можно утверждать, что полученные нами результаты для ФРИ в собственном газе в слабых полях хорошо согласуются в частных случаях с данными других авторов.

На рис. 2 приведены расчеты ФРИ $g_{1}(x)$ и $g_{2}(x)$ без учета упругих столкновений для случаев $\mathrm{He}^{+}-\mathrm{He}$ и $\mathrm{Xe}^{+}-\mathrm{Xе.} \mathrm{При} \mathrm{расчетах} \mathrm{для} \mathrm{сечения} \mathrm{резонансной} \mathrm{пе-}$ резарядки использовалась формула (11) с данными [18]. Аналогично предыдущему видно, что второе приближение практически совпадает с первым. Кроме того, из этих результатов следует, что вид $g(x)$ слабо зависит от сечения перезарядки.

Далее мы провели расчеты зависимости приведенной подвижности $K_{0}=\frac{P[\mathrm{Torr}]}{760} \frac{273.16}{T_{a}[\mathrm{~K}]} \mathrm{K}$ ионов $\mathrm{He}^{+}$и $\mathrm{Ar}^{+}$при нулевом поле в собственных газах от температуры газа в диапазоне от криогенных до $1000 \mathrm{~K}$. Эти газы были выбраны, поскольку для них имеются надежные данные по сечениям упругого рассеяния в широком диапазоне относительной энергии атома и иона $[5,19,20]$. Результаты для $\mathrm{He}^{+}$и $\mathrm{Ar}^{+}$приведены на рис. 3 и 4 соответственно. На рис. 3 приведены экспериментальные данные из работ [21-27] и результаты расчетов авторов [28,29]. Как показано в [21], зависимость приведенной подвижности $\mathrm{He}^{+}$в Не от температуры газа можно получить из зависимости этой величины от параметра $E / N$, если для температуры газа использовать эффективную температуру $T_{\text {eff, }}$ которую для случая Не следует рассчитывать по формуле

$$
T_{\mathrm{eff}}=T_{a}+2.9 \cdot 10^{-4} m_{\mathrm{He}}\left(K_{0} \frac{E}{N}\right)^{2},
$$

где $T_{\text {eff }}, T_{a}$ выражены в градусах Кельвина, $m_{\mathrm{He}}-$ масса атома Не в атомных единицах; приведенная подвижность $K_{0}-$ в $\mathrm{cm}^{2} / \mathrm{Vc}, E / N-$ в Td. Экспериментальные данные из [21] по зависимости подвижности $\mathrm{He}^{+}$в Не от параметра $E / N$, пересчитанные по формуле (14), также приведены на рис. 3. Здесь же показаны результаты наших расчетов при параметре $\sigma=0.31$ для сечения резонансной перезарядки, согласно (11), с параметрами, взятыми из [18], и поправкой на отклонение траекторий сталкивающихся частиц от прямых при малых относительных энергиях иона и атома в виде множителя $\left[1+\frac{V\left(R_{0}\right)}{\varepsilon}\right]$, где $R_{0}=\sqrt{\frac{2 \sigma_{c}}{\pi}}$ [30]. Это необходимо, поскольку расчеты проводились в том числе и для криогенных температур, при которых эта поправка может играть заметную роль. Кроме того, учитывалось, что при низких энергиях относительного движения сечение резонансной перезарядки становится равным половине сечения поляризационного захвата [30]. Сечение переноса энергии, приведенное в [29], хорошо аппроксимируется формулой

$$
\sigma_{v}(\varepsilon)=\frac{\sigma_{m}(\varepsilon)}{1.5\left(1+\varepsilon^{1.1}\right)}, \quad \sigma_{m}(\varepsilon)=2 \sigma_{c}(\varepsilon)
$$

Из этих соотношений и (4) следует, что $\sigma=1$. Однако значение этого параметра, найденное из условия наилучшего совпадения с экспериментальными данными, как уже указывалось, равно $\sigma=0.31$. Это можно объяснить тем, что, как следует из экспериментальных данных работы [20], дифференциальное сечение упругого рассеяния иона $\mathrm{He}^{+}$на атоме Не при энергиях порядка $1 \mathrm{eV}$ имеет при малых углах рассеяния ярко выраженный максимум. Это обстоятельство увеличивает интегральное сечение упругого рассеяния, но при этом практически не оказывает влияния на подвижность иона, поскольку соответствует рассеянию с малыми изменениями импульса 


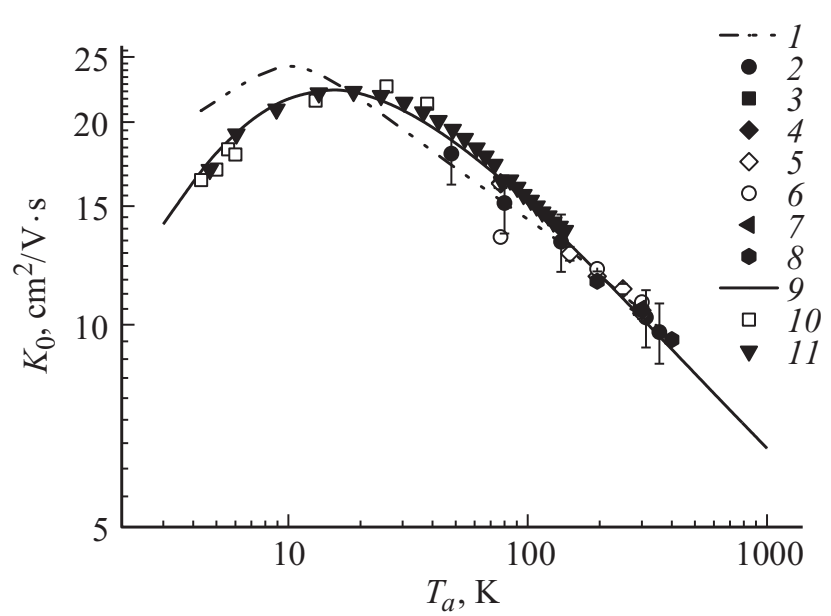

Рис. 3. Зависимости подвижности $K_{0}$ иона $\mathrm{He}^{+}$в Не от температуры атомов: 1 - расчет [28], 2 - эксперимент [21], 3 эксперимент [22], 4 - расчет [29], 5 - эксперимент [23], 6 эксперимент [24], 7 - эксперимент [25], 8 - эксперимент [26], 9 - расчет по полученным формулам с использованием сечений рассеяния для Не из $[18,29]$ при $\sigma=0.31,10-$ пересчет данных [29] по $T_{\text {eff }}$ (см. формулу (14)), 11 пересчет данных [21] по $T_{\text {eff }}$ (см. формулу (14)).

и энергии иона. Таким образом, при аппроксимации дифференциального сечения рассеяния иона на собственном атоме, согласно (1) (т. е. без учета максимума при малых углах рассеяния), следует брать интегральное сечение рассеяния меньше реального. Именно этот факт и объясняет, почему найденное из лучшего совпадения расчета с экспериментом отношение сечений упругого рассеяния и резонансной перезарядки при $1 \mathrm{eV}$ меньше, чем полученное из квантово-механических расчетов [29].

На рис. 4 приведены данные по приведенной подвижности для $\mathrm{Ar}^{+}$в $\mathrm{Ar}$ при нулевом поле, рассчитанные для различных сечений перезарядки. Расчеты проводились для сечения резонансной перезарядки, взятого из [18], и сечения упругого рассеяния из [30] (кривая 3 на рис. 4). В другом варианте использовалось сечение резонансной перезарядки, которое определялось по соотношению (3) с сечением переноса импульса $\sigma_{m}$ и сечением упругого рассеяния из [31] (кривая 1 на рис. 4). При этом параметр $\sigma$ в обоих случаях не варьировался, а вычислялся с использованием известных сечений $[18,31]$ и равнялся $\sigma=0.514,0.488$ соответственно. Здесь же приведены результаты расчетов с использованием тех же сечений резонансной перезарядки, но без учета упругого рассеяния. Из приведенных данных видно, что, поскольку сечение резонансной перезарядки, приведенное в [18], превосходит вычисленное из данных [31] по $\sigma_{m}$, кривая 3 расположена заметно выше кривой 1. Кроме того, в области низких температур (при $T_{a}<30 \mathrm{~K}$ ) вычисленные подвижности по-разному зависят от температуры атомов. Это также, по-видимому, связано с разной температурной зависимостью приведенных в [18] и рассчитанных с помощью диффузионного сечения из [31] сечений перезарядки при низких относительных энергиях иона и атома. Если говорить о соответствии расчетов экспериментальным данным, то в целом расчеты по [31] лучше согласуются с ними. Хотя следует отметить значительное отличие данных по подвижности ионов $\mathrm{Ar}^{+}$в $\mathrm{Ar}$ у разных авторов [11,24,32-36].

Необходимо также подчеркнуть, что, как видно из приведенных на рис. 4 кривых, пренебрежение упругим рассеянием приводит к существенной погрешности при расчете подвижности при температурах ниже $300 \mathrm{~K}$, и эта погрешность увеличивается при уменьшении температуры атомов. Ясно, что причина этого - более резкий рост сечения упругого рассеяния при уменьшении относительной энергии иона и атома (которая при нулевом поле определяется температурой атомов) по сравнению с сечением резонансной перезарядки. Следует также отметить, что если при расчете подвижности $\mathrm{He}^{+}$в Не для наилучшего совпадения с экспериментом нам пришлось выбрать параметр $\sigma$ меньшим, чем отношение использованных упругого сечения и сечения резонансной перезарядки (причина объяснена выше), то в случае с аргоном этого не потребовалось, и параметр $\sigma$ был взят из отношения сечений. Причина такого различия в том, что, как следует из сравнения данных по дифференциальным сечениям упругого рассеяния иона $\mathrm{Ar}^{+}$на атоме $\mathrm{Ar}[3]$ и иона $\mathrm{He}^{+}$на атоме $\mathrm{He}$ [20], при энергии относительного движения иона и атома около $1 \mathrm{eV}$ (при которой и вычисляется параметр $\sigma$ ), в первом случае сечение близко к изотропному в системе центра масс, в то время как во втором - имеется существенный максимум при малых углах рассеяния. Последнее обстоятельство, как мы уже говорили, умень-

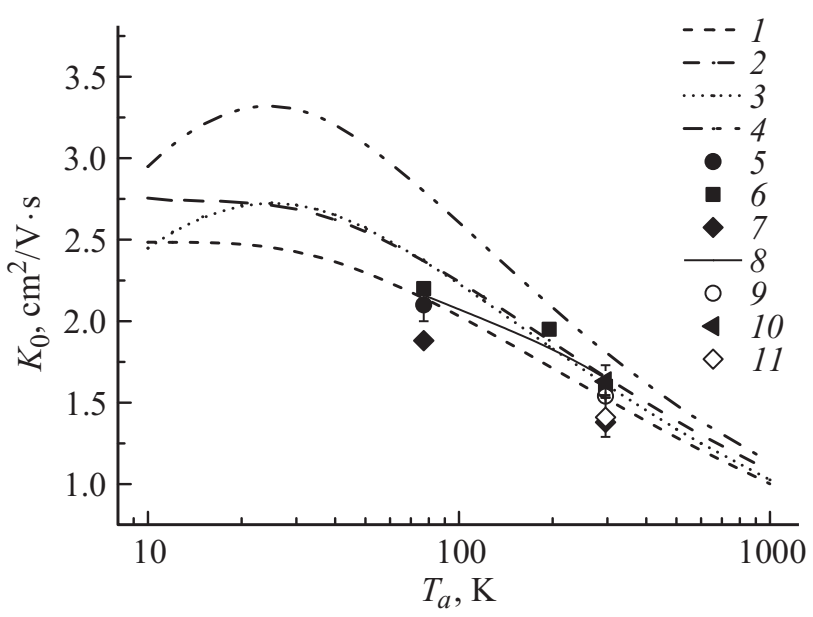

Рис. 4. Зависимости подвижности $K_{0}$ иона $\mathrm{Ar}^{+}$в $\mathrm{Ar}$ от температуры атомов: 1 - расчеты по полученным формулам с использованием сечения перезарядки и упругого рассеяния из [31] при $\sigma=0.488,2-$ то же, что и 1 , но при $\sigma=0,3-$ расчеты по полученным формулам с использованием сечения перезарядки из [18], упругого рассеяния из [31] при $\sigma=0.514$, 4 - то же, что и 3, но при $\sigma=0,5$ - эксперимент [32], 6 - эксперимент [24] 7 - эксперимент [33], 8- расчет [36], 9 - эксперимент [34], 10 - эксперимент [11], 11 - эксперимент [35]. 


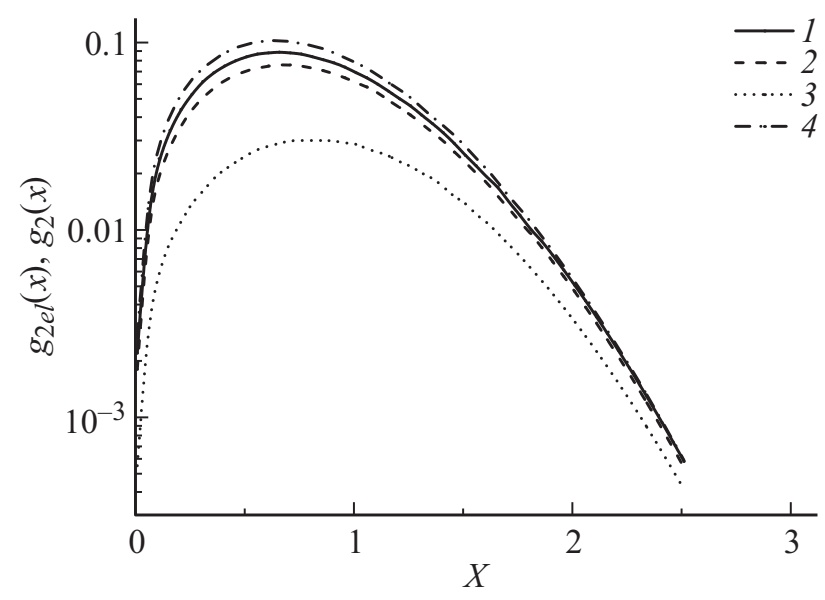

Рис. 5. Влияние упругих столкновений на вид возмущения ФРИ в слабом поле для случая $\mathrm{He}^{+}-\mathrm{He}$ при различной атомной температуре и $\sigma=0.31 .1-g_{e l}(x)-$ второе приближение с учетом упругих столкновений $T_{a}=1000 \mathrm{~K}, 2-$ $g_{e l}(x)-$ второе приближение с учетом упругих столкновений $T_{a}=76 \mathrm{~K}, 3-g_{e l}(x)-$ второе приближение с учетом упругих столкновений $T_{a}=10 \mathrm{~K}, 4-g_{2}(x)-$ второе приближение без учета упругих столкновений. Сечения резонансной перезарядки и упругого рассеяния взяты из [18], [29].

шает вклад изотропного рассеяния иона $\mathrm{He}^{+}$, что и приводит к необходимости уменьшения параметра $\sigma$, поскольку упругое рассеяние на малые углы не влияет на подвижность иона.

На рис. 5 приведены результаты расчета функции $g_{2}(x)$ без учета упругих столкновений для случая $\mathrm{He}^{+}-$ Не и функции $g_{2 e l}(x)$, вычисленной с их учетом для случая $\mathrm{He}^{+}-\mathrm{He}$ при разных температурах газа $T_{a}=10$, $76,1000 \mathrm{~K}$ при параметре $\sigma=0.31$. Как и следовало ожидать, учет упругих столкновений уменьшает поправку ФРИ к максвеловской функции, вызванную наличием электрического поля и процессом резонансной перезарядки, поскольку ведет, во-первых, к изотропизации ФРИ, во-вторых — к дополнительному обмену энергией между атомами и ионами. Кроме того, в отличие от функции $g_{2}(x)$ (рассчитанной с учетом только резонансной перезарядки), которая слабо зависит от температуры газа, влияние на ФРИ $g_{2 e l}(x)$ упругих столкновений растет с уменьшением температуры. В рассматриваемом случае $\mathrm{He}^{+}-\mathrm{He}$ при температуре $10 \mathrm{~K} g_{2 e l}(x)$ падает приблизительно в 3 раза по сравнению с поправкой, рассчитанной без учета упругих столкновений. Это, по-видимому, обусловлено тем, что при низких температурах отношение сечения упругого рассеяния и резонансной перезарядки заметно возрастает.

Таким образом, можно утверждать, что значения подвижности ионов в собственном газе при низких температурах являются чувствительными к отношению сечений упругого рассеяния и резонансной перезарядки, и полученные формулы можно использовать для нахождения энергетической зависимости этого отношения из экспериментальных данных.

\section{Заключение}

В заключение перечислим основные результаты работы:

- получено выражение для ФРИ в слабых полях в собственном газе, которое наряду с процессом резонансной перезарядки учитывает упругие столкновения ионов с атомами. Показано, что упругие столкновения приводят к уменьшению отклонения ФРИ от максвелловской;

- расчеты дрейфовой скорости и подвижности, выполненные по полученным формулам в пренебрежении упругими столкновениями, хорошо согласуются с теоретическими вычислениями других авторов;

- проведены расчеты температурных зависимостей подвижностей ионов $\mathrm{He}^{+}$и $\mathrm{Ar}^{+}$в собственных газах в пределе нулевого поля для диапазона температур газа от 4 до $1000 \mathrm{~K}$, которые хорошо описывают имеющиеся экспериментальные данные. В отличие от случая сильных и умеренных полей пренебрежение упругими столкновениями иона с атомом приводит к существенному завышению рассчитанной подвижности, особенно при криогенных температурах;

- по результатам сравнения расчетных и экспериментальных подвижностей при низких температурах можно восстановить отношения сечений упругого рассеяния иона на атоме и резонансной перезарядки.

\section{Приложение}

Уравнение (11b) представляет собой сингулярно возмущенное интегродифференциальное уравнение с малым параметром $\varepsilon_{0}$ при старшей производной [16]. Доказательство близости его приближенного решения, полученного путем разложения по малому параметру, требует отдельного исследования. Поэтому для нахождения этого решения применим известный метод многих масштабов [17], который используется для решения сингулярно возмущенных уравнений. Вводя вместо переменной $x$ переменные $x_{1}=x, \tau=x / \varepsilon_{0}$, получим интегродифференциальное уравнение, которое регулярно по малому параметру $\varepsilon_{0}$, и его решение можно искать в виде разложения в степенной ряд по этому малому параметру. Принимая во внимание, что $\frac{\partial}{\partial x}=\frac{\partial}{\partial x_{1}}+\varepsilon_{0}^{-1} \frac{\partial}{\partial \tau}$, для первых двух членов этого разложения получим

$$
f_{i}=F_{0}\left(x_{1}, \tau, \mu\right)+\varepsilon_{0} F_{1}\left(x_{1}, \tau, \mu\right),
$$

$$
\begin{aligned}
& \mu \frac{\partial F_{0}}{\partial \tau}+2 \tilde{w}_{o e}\left(x_{1}\right)\left[k\left(x_{1}\right)+0.5 \sigma k_{e}\left(x_{1}\right)\right] F_{0} \\
& \quad=2\left[k(x)-0.5 \sigma k_{e}(x)\right] f_{a}\left(x_{1}\right) \int x_{r} F_{0}\left(\mathbf{x}_{1}^{\prime}, \tau\right) d \mathbf{x}_{1}^{\prime} \\
& \quad+2 \sigma \int k_{e}\left(x^{\prime}\right) \tilde{w}_{e}\left(\mathbf{x}_{1}^{\prime} \rightarrow \mathbf{x}_{1}\right) F_{0}\left(\mathbf{x}_{1}^{\prime}, \tau\right) d \mathbf{x}_{1}^{\prime},
\end{aligned}
$$




$$
\begin{aligned}
& \mu \frac{\partial F_{0}}{\partial x_{1}}+\mu \frac{\partial F_{1}}{\partial \tau}+2 \tilde{w}_{o e}\left(x_{1}\right)\left[k\left(x_{1}\right)+0.5 \sigma k_{e}\left(x_{1}\right)\right] F_{1} \\
& \quad=2\left[k(x)-0.5 \sigma k_{e}(x)\right] f_{a}\left(x_{1}\right) \int x_{r} F_{1}\left(\mathbf{x}_{1}^{\prime}, \tau\right) d \mathbf{x}_{1}^{\prime} \\
& \quad+2 \sigma \int k_{e}\left(x^{\prime}\right) \tilde{w}_{e}\left(\mathbf{x}_{1}^{\prime} \rightarrow \mathbf{x}_{1}\right) F_{1}\left(\mathbf{x}_{1}^{\prime}, \tau\right) d \mathbf{x}^{\prime} .
\end{aligned}
$$

Для решения системы (П1), воспользовавшись тем, что при слабых полях ФРИ близка к изотропной, применим известный $P_{1}$-метод [14]:

$$
F_{i}=\frac{1}{4 \pi}\left(F_{i 0}+3 \mu F_{i 1}\right), \quad i=0,1 .
$$

Подставляя это разложение в (П1) и интегрируя по углам, затем умножая на $\mu$ и вновь интегрируя по углам, получаем систему уравнений для функций $F_{i k}\left(x_{1}, \tau\right)$, $i, k=0,1$ :

$$
\begin{aligned}
& \frac{\partial F_{01}}{\partial \tau}+2 \tilde{w}_{o e}\left(x_{1}\right)\left[k\left(x_{1}\right)+0.5 \sigma k_{e}\left(x_{1}\right)\right] F_{00} \\
& =2\left[k(x)-0.5 \sigma k_{e}(x)\right] \int \alpha_{0 r}\left(x_{1}^{\prime}, x_{1}\right) F_{00}\left(x_{1}^{\prime}, \tau\right) d x_{1}^{\prime} \\
& \quad+2 \sigma \int k_{e}\left(x^{\prime}\right) \alpha_{0}\left(x_{1}^{\prime} \rightarrow x_{1}\right) F_{00}\left(x_{1}^{\prime}, \tau\right) d x_{1}^{\prime}, \\
& \frac{\partial F_{01}}{\partial x_{1}}+\frac{\partial F_{11}}{\partial \tau}+2 \tilde{w}_{o e}\left(x_{1}\right)\left[k\left(x_{1}\right)+0.5 \sigma k_{e}\left(x_{1}\right)\right] F_{10} \\
& =2\left[k(x)-0.5 \sigma k_{e}(x)\right] f_{a}\left(x_{1}\right) \int \alpha_{0 r}\left(x_{1}^{\prime}, x_{1}\right) F_{10}\left(x_{1}^{\prime}, \tau\right) d x_{1}^{\prime} \\
& +2 \sigma \int k_{e}\left(x^{\prime}\right) \alpha_{0}\left(x_{1}^{\prime} \rightarrow x_{1}\right) F_{10}\left(x_{1}^{\prime}, \tau\right) d x_{1}^{\prime}, \\
& \frac{1}{3} \frac{\partial F_{00}}{\partial \tau}+2 \tilde{w}_{o e}\left(x_{1}\right)\left[k\left(x_{1}\right)+0.5 \sigma k_{e}\left(x_{1}\right)\right] F_{01} \\
& =2\left[k(x)-0.5 \sigma k_{e}(x)\right] f_{a}\left(x_{1}\right) \int \alpha_{1 r}\left(x_{1}^{\prime}, x_{1}\right) F_{01}\left(x_{1}^{\prime}, \tau\right) d x_{1}^{\prime} \\
& +2 \sigma \int k_{e}\left(x^{\prime}\right) \alpha_{1}\left(x_{1}^{\prime} \rightarrow x_{1}\right) F_{01}\left(x_{1}^{\prime}, \tau\right) d x_{1}^{\prime}, \\
& \frac{1}{3} \frac{\partial F_{00}}{\partial x_{1}}+\frac{1}{3} \frac{\partial F_{10}}{\partial \tau}+2 \tilde{w}_{o e}\left(x_{1}\right)\left[k\left(x_{1}\right)+0.5 \sigma k_{e}\left(x_{1}\right)\right] F_{11} \\
& =2\left[k(x)-0.5 \sigma k_{e}(x)\right] f_{a}\left(x_{1}\right) \int \alpha_{1 r}\left(x_{1}^{\prime}, x_{1}\right) F_{11}\left(x_{1}^{\prime}, \tau\right) d x_{1}^{\prime} \\
& +2 \sigma \int k_{e}\left(x^{\prime}\right) \alpha_{1}\left(x_{1}^{\prime} \rightarrow x_{1}\right) F_{11}\left(x_{1}^{\prime}, \tau\right) d x_{1}^{\prime},
\end{aligned}
$$

где

$$
\begin{aligned}
& \alpha_{0 r}\left(x_{1}^{\prime}, x_{1}\right)=2 \pi \int_{-1}^{1} \sqrt{x_{1}^{\prime 2}+x_{1}^{2}-2 x_{1}^{\prime} x_{1} \mu} d \mu, \\
& \alpha_{1 r}\left(x_{1}^{\prime}, x_{1}\right)=2 \pi \int_{-1}^{1} \mu \sqrt{x_{1}^{\prime 2}+x_{1}^{2}-2 x_{1}^{\prime} x_{1} \mu} d \mu,
\end{aligned}
$$

$$
\begin{gathered}
\alpha_{0}\left(x_{1}^{\prime} \rightarrow x_{1}\right)=2 \pi \int_{-1}^{1} \tilde{w}_{e}\left(\mathbf{x}_{1}^{\prime} \rightarrow \mathbf{x}_{1}\right) d \mu, \\
\alpha_{1}\left(x_{1}^{\prime} \rightarrow x_{1}\right)=2 \pi \int_{-1}^{1} \mu \tilde{w}_{e}\left(\mathbf{x}_{1}^{\prime} \rightarrow \mathbf{x}_{1}\right) d \mu .
\end{gathered}
$$

Для $\alpha_{1}\left(x_{1}^{\prime} \rightarrow x_{1}\right)$ справедливы выражения [13]

$$
\begin{gathered}
\alpha_{1}\left(x_{1}^{\prime} \rightarrow x_{1}\right)=2 \frac{\exp \left(x_{1}^{\prime 2}-x_{1}^{2}\right)}{x_{1}^{\prime}} \psi\left(x_{1}^{\prime}\right) \text { при } x_{1}>x_{1}^{\prime}, \\
\alpha_{1}\left(x_{1}^{\prime} \rightarrow x_{1}\right)=2 \frac{x_{1}}{x_{1}^{\prime 2}} \psi\left(x_{1}\right) \quad \text { при } \quad x_{1}<x_{1}^{\prime}, \\
\psi(x)=\frac{2}{\sqrt{\pi}} \exp \left(-x^{2}\right)+\left(x-\frac{1}{x}\right) \operatorname{erf}(x),
\end{gathered}
$$

а для $\alpha_{1 r}\left(x_{1}^{\prime}, x_{1}\right)$

$$
\begin{gathered}
\alpha_{1 r}\left(x_{1}^{\prime}, x_{1}\right)=\frac{2 \pi}{15 \eta^{2}}\left\{\left(1+\eta^{2}-3 \eta\right)\left(1+\eta^{2}+2 \eta\right)^{1.5}\right. \\
\left.-\left(1+\eta^{2}+3 \eta\right)\left(1+\eta^{2}-2 \eta\right)^{1.5}\right\}, \\
\eta=\frac{x_{1}^{\prime}}{x_{1}} .
\end{gathered}
$$

Используя $P_{2}$-приближение [14], можно показать, что выполняется

$$
F_{01}, F_{10} \equiv 0, \quad F_{00}\left(x_{1}, \tau\right)=f_{a}\left(x_{1}\right) .
$$

Тогда, возвращаясь к переменной $x$, получим

$$
\begin{aligned}
& \frac{1}{3} \frac{\partial F_{00}}{\partial x}+2 \tilde{w}_{o e}(x)\left[k(x)+0.5 \sigma k_{e}(x)\right] F_{11} \\
& =2\left[k(x)-0.5 \sigma k_{e}(x)\right] f_{a}(x) \int \alpha_{1 r}\left(x^{\prime}, x\right) F_{11}\left(x^{\prime}\right) d x^{\prime} \\
& +2 \sigma \int k_{e}\left(x^{\prime}\right) \alpha_{1}\left(x^{\prime} \rightarrow x\right) F_{11}\left(x^{\prime}\right) d x^{\prime},
\end{aligned}
$$

Для решения уравнения (П3) рассмотрим сначала случай, когда $\sigma=0$. В этом случае можно применить метод последовательных приближений, который уже применялся в работе [2]:

$$
\begin{aligned}
& F_{11}^{(n)}(x)_{\sigma=0}= \frac{x \exp \left(-x^{2}\right)}{3 \pi^{1.5} \tilde{w}_{o e}(x) k(x)}+2 f_{a}(x) \int \alpha_{1 r}\left(x^{\prime}, x\right) \\
& \times F_{11}^{(n-1)}\left(x^{\prime}\right)_{\sigma=0} d x^{\prime} \\
& F_{11}^{(0)}(x)_{\sigma=0}=\frac{x \exp \left(-x^{2}\right)}{3 \pi^{1.5} \tilde{w}_{o e}(x) k(x)}
\end{aligned}
$$

Расчеты показывают, что второе приближение отличается от первого на величину порядка $1 \%$. Совершенно 
аналогично получается решение при $\sigma \neq 0$. Тогда, используя второе приближение для $F_{11}(x)_{\sigma=0}$, получим

$$
\begin{gathered}
F_{11}^{(n)}(x)=\frac{F_{11}^{(2)}(x)_{\sigma=0}}{1+0.5 \sigma \Omega(x)}+\frac{\sigma[1-0.5 \sigma \Omega(x)]}{\tilde{w}_{o e}(x)[1+0.5 \sigma \Omega(x)]} \\
\quad \times \int_{-1}^{1} k_{e}\left(x^{\prime}\right) \alpha_{1}\left(x_{1}^{\prime} \rightarrow x_{1}\right) F_{11}^{(n-1)}(x) d x \\
F_{11}^{(0)}(x)=\frac{F_{11}^{(2)}(x)_{\sigma=0}}{1+0.5 \sigma \Omega(x)}, \quad \Omega(x)=\frac{k_{e}(x)}{k(x)},
\end{gathered}
$$

где $\alpha_{1}\left(x_{1}^{\prime} \rightarrow x_{1}\right)$ определена соотношениями (П2). Аналогично предыдущему второе приближение данного метода последовательных приближений дает практически точный результат.

\section{Список литературы}

[1] Мустабаев А.С., Сухомлинов В.С., Аинов М.А. // ЖТФ. 2015. Т. 85. Вып. 12. С. 45-56.

[2] Мустафаев А.С., Некучаев В.О., Сухомлинов В.С. // ТВТ. 2016. В печати.

[3] Barata J.A.S. // Nucl. Instrum. Meth. Phys. Res. 2007. P. 14 17.

[4] Barata J.A.S., Conde C.A.N. // Nucl. Instrum. Meth. Phys. Res. 2010. P. 21-23.

[5] Phelps A.V. // J. Appl. Phys. 1994. Vol. 76. P. 747-753.

[6] Смирнов Б.М. // ЖТФ. 1966. Т. 36. Вып. 10. С. 1864-1871.

[7] Хаксли Л., Кромптон Р. Атомные и молекулярные процессы. М.: Мир, 1964. 297 с.

[8] Каган Ю.М., Перель В.И. // ДАН СССР. 1954. Т. 98. C. $575-578$.

[9] Перель В.И. // ЖЭТФ. 1957. Т. 32. С. 526-533.

[10] Фок В.А. // ЖЭТФ. 1948. Т. 18. С. 1048 - 1055.

[11] Мак-Даниель И., Мезон Э. Подвижность и диффузия ионов в газах. М.: Мир, 1976. 422 с.

[12] Чепмен C., Куалинг T. Математическая теория неоднородных газов. М.: ИЛ., 1960. $510 \mathrm{c.}$

[13] Vahedi V., Surendra M. // Comp. Phys. Communic. 1995. Vol. 87. P. 179-198.

[14] Марчук Г.И. Методы расчета ядерных реакторов. М.: Гос. изд-во лит-ры в области атомной науки и техники, 1981. $667 \mathrm{c}$.

[15] Lampe M., Röcker T.B., Joyce G., Zhdanov S.K., Ivlev A.V., Morfill G.E. // Phys. Plasm. 2012. N 19. P. 113703.

[16] Ломов С.A. Введение в общую теорию сингулярных возмущений. М.: Наука, 1981. 398 с.

[17] Найбэ А.Х. Методы возмущений. М.: Наука, 1976. 474 с.

[18] Maiorov S.A. // 28th ICPIG. 2007. Prague, Czech Republic. N 1. P. 77-180.

[19] Vestal M.L., Blakley R., Futrell J.H. // Phys. Rev. 1978. Vol. 17. N 4. P. 1337-1342.

[20] Vestal M.L., Blakley R., Futrell J.H. // Phys. Rev. 1978. Vol. 17. N 4. P. 1321-1336.

[21] Bohringer H., Glebe W., Arnold F. // J. Phys. B. At. Mol. Phys. 1983. Vol. 16. P. 2619-2626.

[22] Skullerud H.R., Larsen P.H. // J. Phys. B. At. Mol. Opt. Phys. 1990. Vol. 23. P. 1017-1041.
[23] Patterson P.L. // Phys. Beview. 1970. Vol. 2. N 4. P. 1154 1164.

[24] Chanin M., Biondi A. // Phys. Beview. 1957. Vol. 106. N 3. P. 473-479.

[25] Helm H. // J. Phys. B. At. Molec. Phys. 1977. Vol. 10. N 18. P. 3683-3697.

[26] Orient O.J. // Can. J. Phys. 1967. Vol. 45. P. 3915-3922.

[27] Saito N., Kojima T.M., Kobayashi N., Kaneko Y. // J. Chem. Phys. 1994. Vol. 100. P. 5726-5734.

[28] Dickinson A.S. // J. Phys. B. At. Mol. Phys. 1968. Vol. 1. P. $387-394$

[29] Dickinson A.S., Lee M.S., Viehland L.A. // J. Phys. B. At. Mol. Opt. Phys. 1999. Vol. 32. P. 4919-4930.

[30] Смирнов Б.М. // УФН. 1962. Т. 92. Вып. 1. С. 75-103.

[31] Phelps A.V. // J. Appl. Phys. 1994. Vol. 76. P. 747-753.

[32] Helm H., Elford M.T. // J. Phys. B. Atom. Molec. Phys. 1978. Vol. 11. N 22. P. 3939-3950.

[33] Beaty E.C. // Phys. Rev. 1956. Vol. 104. P. 17-20.

[34] Beaty E.C. // Proc. 5th Intern. Conf. Ionization Phenomena Gases. Amsterdam: North Holland, 1962. Vol. 1. P. 183-188.

[35] Smith D., Dean A.G., Adams N.G. // Z. Phys. 1972. Vol. 253. P. 191-199.

[36] Holstein T.D. // J. Chem. Phys. 1952. Vol. 56. P. 832. 